\title{
Effect of Reynolds Number on a Plunging Airfoil
}

\section{Diana Carvalho Rodrigues, Emanuel António Rodrigues Camacho, Fernando Manuel da Silva Pereira Neves, André Resende Rodrigues Silva, and Jorge Manuel Martins Barata}

Universidade da Beira Interior

\section{Abstract}

Biomimetics is an area of science that studies the development of new technologies, whose source of inspiration is Nature. Unlike traditional aircraft, animals only have one structure to create both lift and thrust, and for Humans, although in the recent years the studies in this area increased, a long way must be made to achieve their capability. The present paper focuses on the effect of the Reynolds number on the wake configuration produced by a plunging airfoil. The experimental work was performed using an airstream, that was marked with smoke, with an oscillating airfoil NACA0012, whose dimensions are $44 \mathrm{~cm}$ and $10 \mathrm{~cm}$ of span and aerodynamic chord, respectively. Diana Carvalho Rodrigues a33541@ubi.pt

Received: 26 November 2019 Accepted: 13 May 2020

Published: 2 June 2020

Publishing services provided by Knowledge E

(c) Diana Carvalho Rodrigues et al. This article is distributed under the terms of the Creative Commons Attribution License, which permits unrestricted use and redistribution provided that the original author and source are credited.

Selection and Peer-review under the responsibility of the ICEUBI2019 Conference Committee.
Corresponding Author: The motion prescribed for the wing is harmonic, since it very well represents the type of motion seen in Nature. Frequency and amplitude were maintained, respectively, at $1.2 \mathrm{~Hz}$ and $2.8 \mathrm{~cm}$, and the wind speed range from $0.25 \mathrm{~m} / \mathrm{s}$ to $1.00 \mathrm{~m} / \mathrm{s}$, which represents a nondimensional amplitude of 0.28 , a reduced frequencies of $3.02,1.51$ and 0.75 , and a Strouhal number and a Reynolds number range of, $0.07-0.27$ and 1,500 6,300 , respectively. Results indicate that, with the increase of the Reynolds number, the convection effects become more predominant than diffusion effects, the curvature of the wakes and the maximum effective angle of attack decrease, and time and configuration of vortex shedding change. For $\mathrm{Re}=1,500, \mathrm{St}=0.27$, another relevant conclusion appears; the interaction of the leading-edge vortex with the trailing-edge vortex indicates an improvement of the aerodynamic performance of this system.

Keywords: Biomimetics, Plunging, Airfoil, Vortices, Wakes

\section{Nomenclature}

$\alpha_{e f f}$
$\mu$
$\rho$
$A$
$a$
$c$
$f$
$h$
$k$

Effective angle of attack
Dynamic viscosity
Density
Plunge amplitude
Airfoil vertical acceleration
Aerodynamic chord
Plunge frequency
Nondimensional amplitude
Reduced frequency

$\Delta x$
$R e$
$S t$
$T$
$t$
$U_{\infty}$
$v$
$W$
$y$

Displacement Reynolds number Strouhal number Period time Velocity Airfoil vertical velocity Wake width

Airfoil vertical position 


\section{Introduction}

In aeronautics, birds and insects have been the inspiration onto biomimetic studies, since due to the result of millions of years of evolution, they possess enormous maneuverability that is by very far superior to any man-made aerial vehicle maneuver; however, all-natural wing's movements are extremely complex. Thus, biomimetics contributes to a better understanding of the phenomena observed in nature with the purpose of developing new technologies. Unlike the flight of the nowadays airplanes, propulsion produced by engines and lift produced by wings - which is rather restrictive [1] these animals use the wings to produce both lift and thrust simultaneously. In fact, Nature provided biostructures (wings) to flying animals with the capability to perform sequential adaptations on each moment of a wing stroke cycle in order to adapt to each flight phase, a desirable subject for the huge growth of the researcher's community in this field over recent years [2-6]. Although the flapping system is typically a 3D (three dimensional) subject, frequently different authors consider the 2D (two dimensional) assumption which simplifies the analysis and still represents accurately the effects related to oscillating airfoils.

When it comes to flight subjects, the range of Reynolds number present in nature is located between 100 and 10,000 while in the Boeing 747 case could be up to 2,000,000,000 (Figure 1).

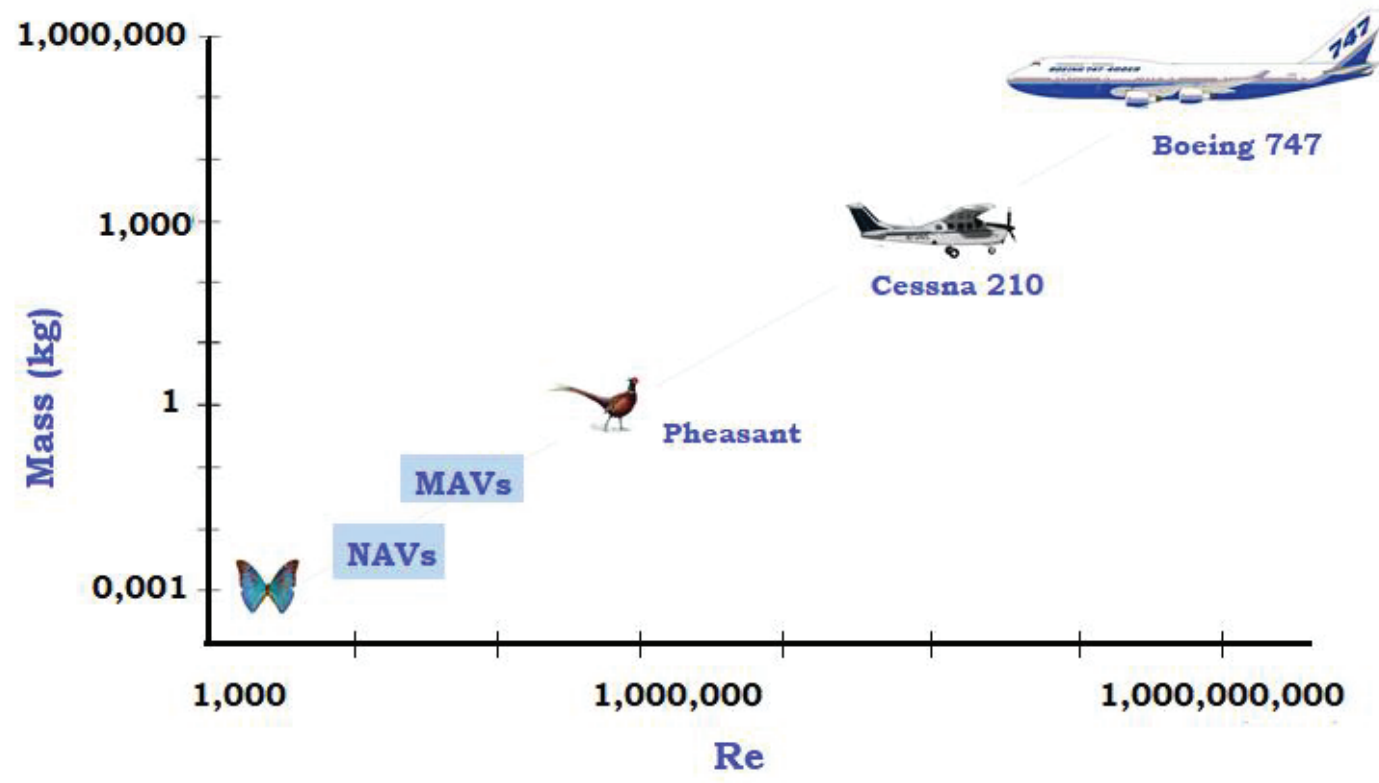

Figure 1: Classification of MAVs and NAVs relatively to Mass (kg) and Reynolds Number (Re) [4]. 
In 1997, DARPA (Defense Advanced Research Projects Agency), initiated a program called "MAV (Micro Aerial Vehicles) project", an initiative in order to seek to develop and to test emerging technologies that could evolve into a mission capable system for military surveillance and reconnaissance applications [7]. The definition employed in DARPA's program limits these vehicles to a size less than $15 \mathrm{~cm}$ in length, width or height, with no restrictions on its design or conception and a maximum total weight less than 100 grams [8]. In October 2005, DARPA launched a Nano Air Vehicle (NAV) program with the objective of developing and demonstrating small ( $<10 \mathrm{~cm}$ wingspan), lightweight ( $<10$ gram) air vehicles systems with the potential to perform challenging indoor and outdoor missions [9].

Knoller and Betz were the first to find that the relative velocity in a flapping airfoil is altered creating an effective angle of attack that gives rise to a normal force to the approaching flow, that can be decomposed in lift and thrust [10, 11].

The flapping motion is typically composed of three motions: the purely vertical motion, denominated by plunging; rotational motion where only the angle of attack is varied, designated by pitching; and, surging, representing the translation motion in the horizontal direction.

Flapping airfoils, more specifically plunging airfoils, have been also studied by Lai \& Platzer [12], Lewin \& Haj-Hariri [13] and Young [14], where wake structures were analyzed, such as the vortex-pair shedding that represent the transition from the drag producing wake (Figure 2 - above) to the thrust producing inverted von Kármán vortex street (Figure 2 - below). In this figure, it is possible to conclude that a von Kármán vortex street can be linked to drag production, while an inverted von Kármán vortex street produces a jet pointing backward, a synonym of thrust. Young \& Lai [15] concluded that this type of wake structure was due to the interaction between bluff-body type natural shedding from the trailing edge and the airfoil motion.

Interested in finding which flying conditions animals fly, Taylor et al [16] studied 42 species (birds, bats and insects) on cruise flight and verified that these animals fly within a limited range of $0.2 \leq S t \leq 0.4$, concluding that this parameter is a possible indicator of the flapping conditions that provide the most efficient flight, being essential to characterize the flight of several natural flyers, regardless of their scale.

To study the flapping airfoil, dimensionless parameters are selected to better understand the effects observed in the flow field, which are presented in Table 1. 


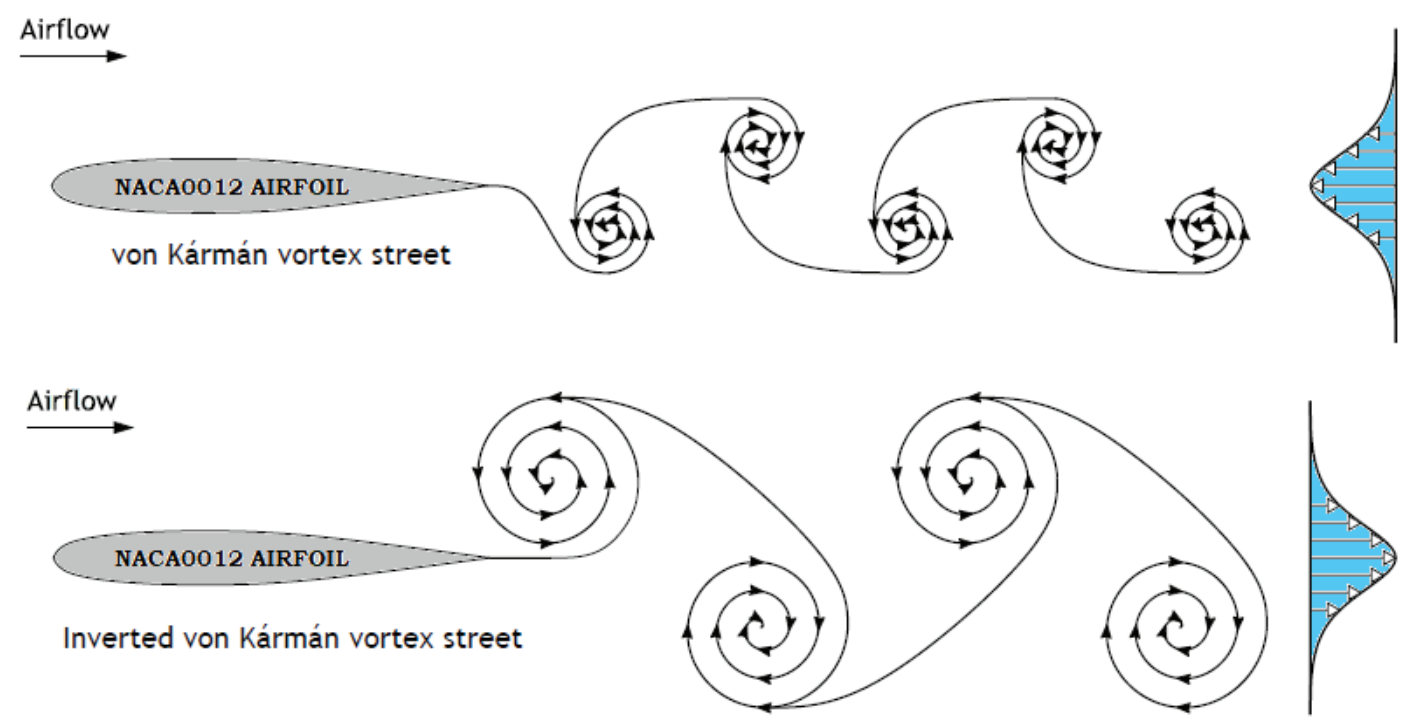

Figure 2: above): Typical von Kármán vortex street - velocity profile indicates a momentum deficit; below): Inverted von Kármán vortex street (velocity profile indicates a momentum surplus) [17].

TABLE 1: Dimensionless Parameters.

\begin{tabular}{c|c|c|c} 
Reynolds Number Re & Reduced Frequency $k$ & $\begin{array}{c}\text { Non-dimensional } \\
\text { Amplitude } h\end{array}$ & Strouhal Number $S t$ \\
\hline $\operatorname{Re}=\frac{\rho U_{\infty} c}{\mu}$ & $k=\frac{2 \pi f c}{U_{\infty}}$ & $h=\frac{A}{c}$ & $S t=\frac{2 f A}{U_{\infty}}$
\end{tabular}

\section{Methodology}

For this experimental work, a NACA0012 airfoil, a common airfoil used in this type of studies, is being used with a span of $44 \mathrm{~cm}$ and an aerodynamic chord of $10 \mathrm{~cm}$, obtained by 3D printing using ABS P430 XL. This airfoil is coupled to an electric motor which has a linear actuator that converts the rotational into translational motion. The motor is an AR series FLEX AC power input built-in controller type of ARM46AC model, and the linear actuator is the EASM4XE005 ARAC model with a $0.006 \mathrm{~mm} / \mathrm{step}$, both produced by Oriental Motors. The airfoil's motion is programmed in the MEXEO2 (Version 3.32.0.0) software, and the information is then transmitted to a controller (ARD-CD model) using a CC051IF-USB cable.

The airfoil's motion implemented follows the uniformly accelerated motion equations described as

$$
\begin{gathered}
y(t)=y_{0}+v_{0} t+\frac{1}{2} a t^{2} \\
v(t)=v_{0}+a t
\end{gathered}
$$


And the effective angle of attack may be calculated as

$$
\alpha_{e f f}(t)=\operatorname{atan}\left(\frac{v(t)}{U_{\infty}}\right)
$$

In Figure 3, the operating speed is shown as a function of time, where the acceleration/deceleration is selected by defining the time that the motor has to accelerate/decelerate. In order to achieve the operating speed (OS) imposed, equation 4 is used which is based on the desired amplitude, $A=\Delta x_{c} / 2=\Delta x_{d} / 2$, and the established motion's period, $T=t_{f}$.

$$
O S=\frac{4 A}{0.5 T}
$$

The airfoil was positioned at $3 \mathrm{~cm}$ of the exit of a wind tunnel $(0.4 \mathrm{~m} \times 0.3 \mathrm{~m})$ and the flow leaving this section was marked recurring to a smoke machine connected to an airfoil placed inside the wind tunnel, that releases smoke at the trailing edge of the airfoil placed vertically with the purposed of minimizing perturbations. The smoke leaving the tunnel was illuminated with a laser sheet, created by a laser placed in the far field that would not disturb the flow field. With the purpose of improving lighting, a focus light was placed on the upper part of the wake created by the airfoil in order to better reflect the smoke particles. The image acquisition was obtained using the Photron FASTCAM mini UX 50 that captured images in a $1280 \times 1024$ resolution, and the frame rate and shutter speed were chosen as $500 \mathrm{fps}$ and $1 / 500$ s, respectively. The low light option was activated, and the camera diaphragm was totally open. These configurations were all selected using the Photron FASTCAM Viewer. Placed at approximately $3.5 \mathrm{~m}$ from the oscillating wing, the camera captured the flow field with a right angle to the laser sheet plane and a small inclination with the horizontal to clearly capture the airfoil's upper surface. The experimental rig is shown in Figure 4. The temperature and ambient pressure were constantly monitored to estimate the fluid properties such as dynamic viscosity, using Sutherland's law, and air density, calculated recurring to the ideal gas law. In the present work, the variable under control is the wind speed, while the motion's frequency and amplitude are kept constant.

\section{Results}

In this section, results are presented and discussed where the influence of the Reynolds number on the flow field created by the NACA0012 oscillating airfoil is studied. The results are obtained for an amplitude of $2.8 \mathrm{~cm}$, a frequency of $1.2 \mathrm{~Hz}$ and a wind speed ranging between $0.25-1.00 \mathrm{~m} / \mathrm{s}$. The studies are then performed with a nondimensional amplitude of 0.28 , reduced frequencies of $3.02,1.51$ and 0.75 and Reynolds numbers of 


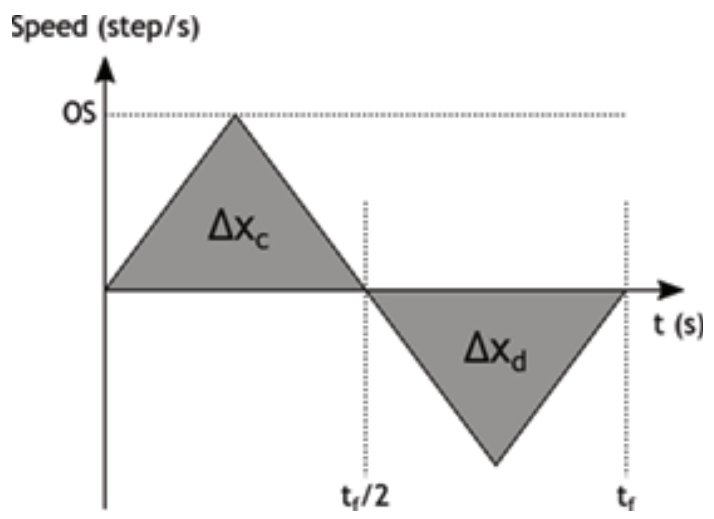

Figure 3: Prescribed motion (1 step $\left./ \mathrm{s}=6 \times 10^{-6} \mathrm{~m} / \mathrm{s}\right)$.

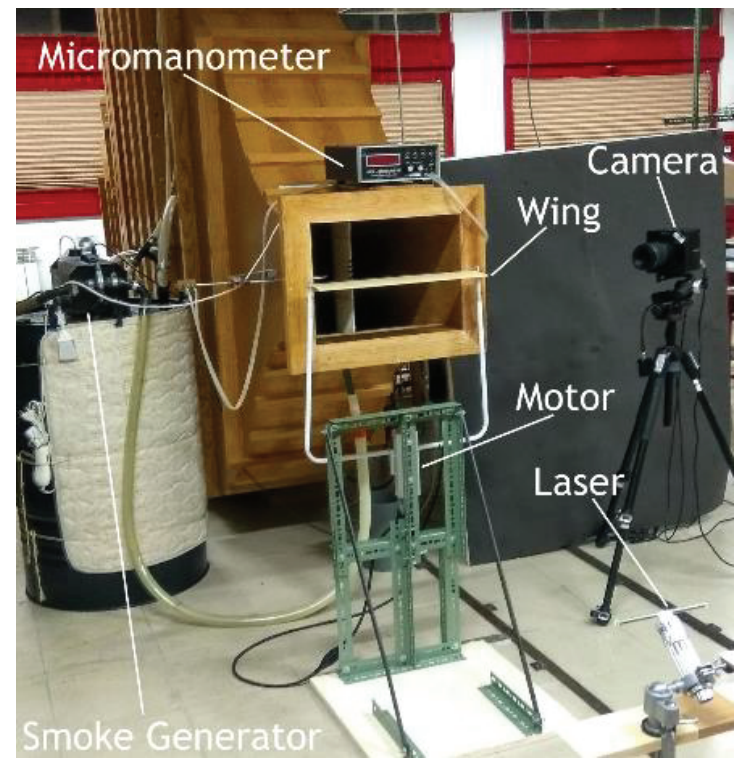

Figure 4: Experimental facility.

approximately 1,500, 3,100 and 6,300. The results are presented in Figure 5 where the flow field is shown as a function of the Reynolds number and fraction of the motion's period. The NACA0012 airfoil is located on the right side of the subfigures and the flow field is moving from the right to the left side.

Beginning with the first case, represented by a $\mathrm{Re} \approx 1,500$, it is clearly seen an inverted von Kármán vortex street that is correlated with thrust production, which comes from the momentum surplus that the wing induces in the airfoil's wake. These vortices work together to originate a series of jets that have an angle with the chordwise direction, that can be projected on this orientation, obtaining a propulsive force, based on Newton's third law of motion.

Regarding the different phases of the motion, and knowing that $t / T=0.00$ represent the lower absolute position during the airfoil's motion, it is observed that a vorticity zone at the trailing- edge starts growing with an anticlockwise rotation and that is 
later shed while the airfoil is moving upwards, until approximately one fourth of the period. Similarly, when the airfoil reaches its maximum absolute coordinate, $t / T=0.50$, a clockwise vorticity region is originated at the trailing-edge, being shed up to about the motion's central position. Due to the periodic essence of the problem, the phenomena repeat itself, however in different configurations because of turbulence effects that are difficult to quantify.

Another singularity of this case is the presence of a leading-edge vortex that surges during the plunging motion, and in these experimental results, it is only seen on the upper surface of the airfoil, though being also observed on the lower surface. The upper leading-edge vortex forms when the airfoil is descending, and it is created due to the effective angle of attack imposed on the airfoil exceeding a critical value, where the boundary layer cannot resist the local adverse pressure gradient and separates. This vortex is later transported by the flow and there is evidence that its vorticity may contribute to the vortices shed downstream, an effect that will be explored later on.

In the second case, the wind speed was increased to $0.5 \mathrm{~m} / \mathrm{s}$ corresponding to a Reynolds number of 3,100. This increment also affects the reduced frequency as well as the Strouhal number, even though, the motion's frequency and amplitude are kept constant. Comparing this condition with the previously analyzed, the effects detected are similar but the flow curvature present on the airfoil's wake is less evident, and the vortex shedding occurs at approximately the minimum and maximum positions of the motion. These effects are associated with the Reynolds number increase that conveys the idea that convection (Convention is related with macroscopic interactions of the fluid associated with its velocity field, in other words, related with the flow inertia effects.), compared to diffusion (Diffusion is related with viscosity effects (microscale effects), where flow properties transmit their information through the interaction of molecules.) effects become more preponderant, and the decrease of the reduced frequency, which clearly reduces the curvature in the wake, shows that the effective angle of attack was also affected. Analogously to the $\mathrm{Re}=1,500$ condition, a leading-edge vortex is detected however with totally different behavior, effect studied in Figure 6 .

The wind speed was further increased to $1.00 \mathrm{~m} / \mathrm{s}$ which rises the convection effects and although imperceptible, a small von Kármán vortex street appears, meaning that the thrust production is compromised. However, the flow field still displays a small curvature which is a synonym of instantaneous lift production caused by the oscillatory effective angle of attack. Returning to the study of the leading-edge vortex effect, and focusing on the $\operatorname{Re}=1,500$, it is possible to analyze the temporal evolution of this structure on the flow field that forms during descent at the leading-edge. Figure 6(a) illustrates the creation 
of an anticlockwise LEV (Leading-Edge Vortex), where the critical effective angle of attack was reached, being later convected in the chordwise direction as observed in subfigures (b) and (c). It is important to mention that the presence of this vortex in the airfoil's upper surface, represents a moving low- pressure zone that contributes to lift production. The hypothesis suggested before, that this vortex could improve the wake's vorticity distribution, can be verified in Figure 6(d), where the anticlockwise is feeding the also anticlockwise vorticity zone shed on the trailing edge of the airfoil. This effect suggests that the leading-edge vortex, linked to flow separation that is typically correlated with drag production, may indeed help thrust generation.

In the case $\operatorname{Re}=3,100$, the LEV formed shows a different behavior since it never leaves the trailing-edge, being dissipated at around fifty percent of the aerodynamic chord. Its formation starts on the leading-edge with a smaller dimension when compared to the previous case and it is clearly seen that its configuration is unstable. In Figure 7(a) it is possible to see the formation of an anticlockwise vortex that is rapidly dissipated over the chordwise direction. In subfigure (c) the vortex already has disappeared, which will not contribute to the anticlockwise vorticity zone shed on trailing-edge of airfoil nor thrust production.

A parallel evaluation must be made between the results presented above and Taylor's observations [16] in order to understand the most efficient way to oscillate the airfoil. Taylor concluded that all animals operate in an interval of Strouhal number between 0.2 and 0.4 and that may indicate the optimal technique to use biostructures to produce an aerodynamic force. In the obtained results, only one condition is in the referred range, which is $R e=1,500$ and a Strouhal number of 0.27 , where a close investigation should be made to further comprehend the effects at the lower Reynolds number tested. Thus, an important conclusion of the present work is that optimal flapping operation at lower Reynolds numbers is achieved with both leading- and trailing-edge vortex shedding.

\section{Conclusion}

Improving current systems based on the phenomena observed in biology is a clear advantage to the scientific community that will exploit the results of a long process of natural selection present in Nature. Understanding the effects of the governing parameters such as wind speed, motion's frequency and amplitude on oscillatory wings is then a clear path to explore new design concepts that may improve aeronautical operations (lower drag production and consequently less fuel consumption), as aircraft manufacturers are currently suggesting. 

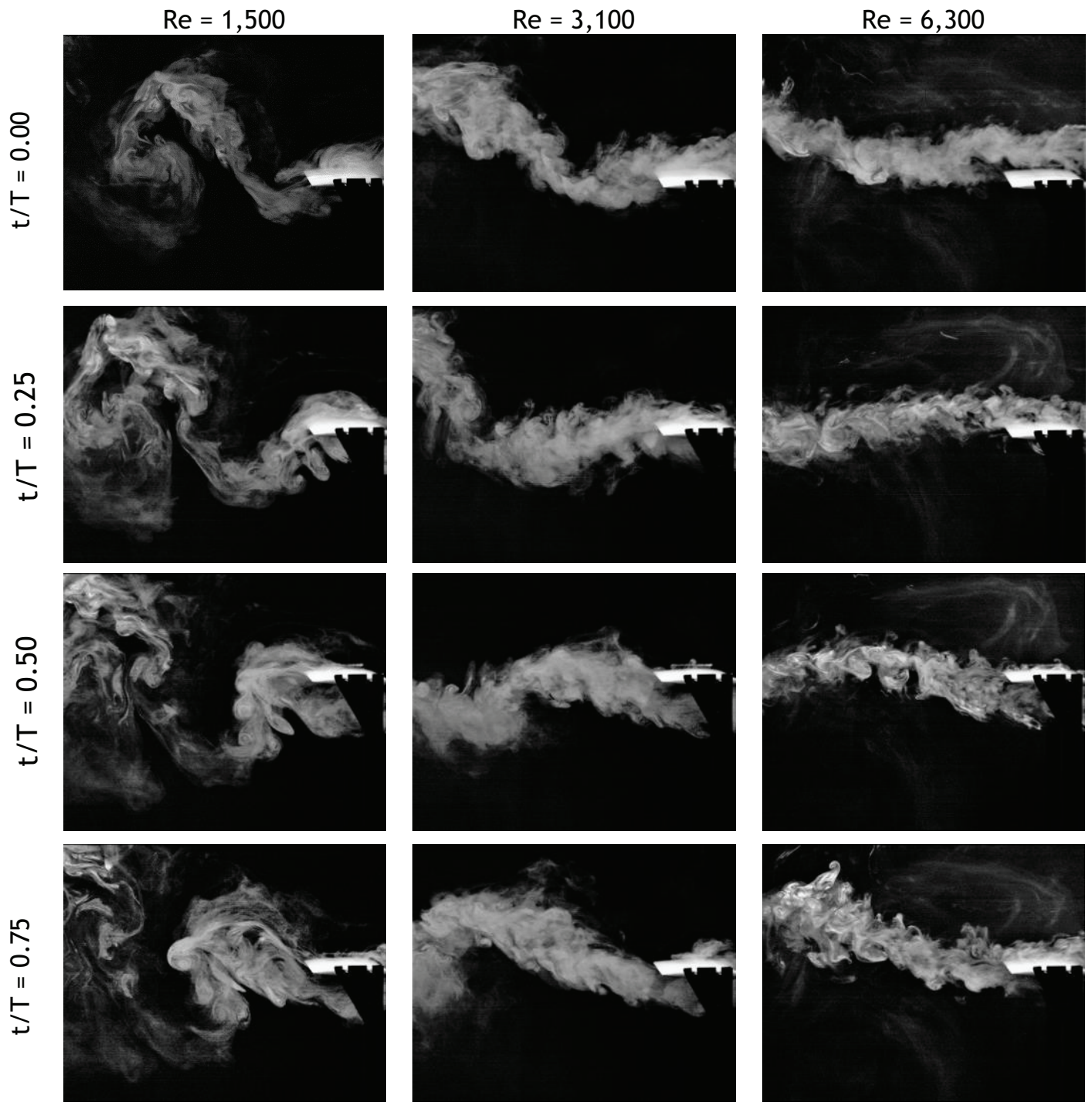

$$
\begin{gathered}
k=3.02 \quad h=0.28 \\
\text { St }=0.27
\end{gathered}
$$

$$
k=1.51 \quad h=0.28
$$$$
\text { St }=0.13
$$

$$
k=0.75 \quad h=0.28
$$$$
\text { St }=0.07
$$

Figure 5: Flow field as a function of Reynolds number and period phase.

The present work focuses exclusively on the influence of the Reynolds number on an oscillatory NACA0012 airfoil subjected to a frequency of $1.2 \mathrm{~Hz}$ and an amplitude of $2.8 \mathrm{~cm}$. The Reynolds number was changed by varying the wind velocity of the wind tunnel, which directly affected the reduced frequency but kept the nondimensional amplitude constant. Thus, the Strouhal number ranges between 0.07-0.27 which intersects with the typical regime that animals operate in.

Results indicate that considering the flapping condition that the airfoil was tested, the Reynolds number has an enormous influence in the flow field created by the oscillating airfoil, an influence that comes from the relative importance of convection and diffusion effects. Thus, the Reynolds number changed the vortex shedding configuration of the 

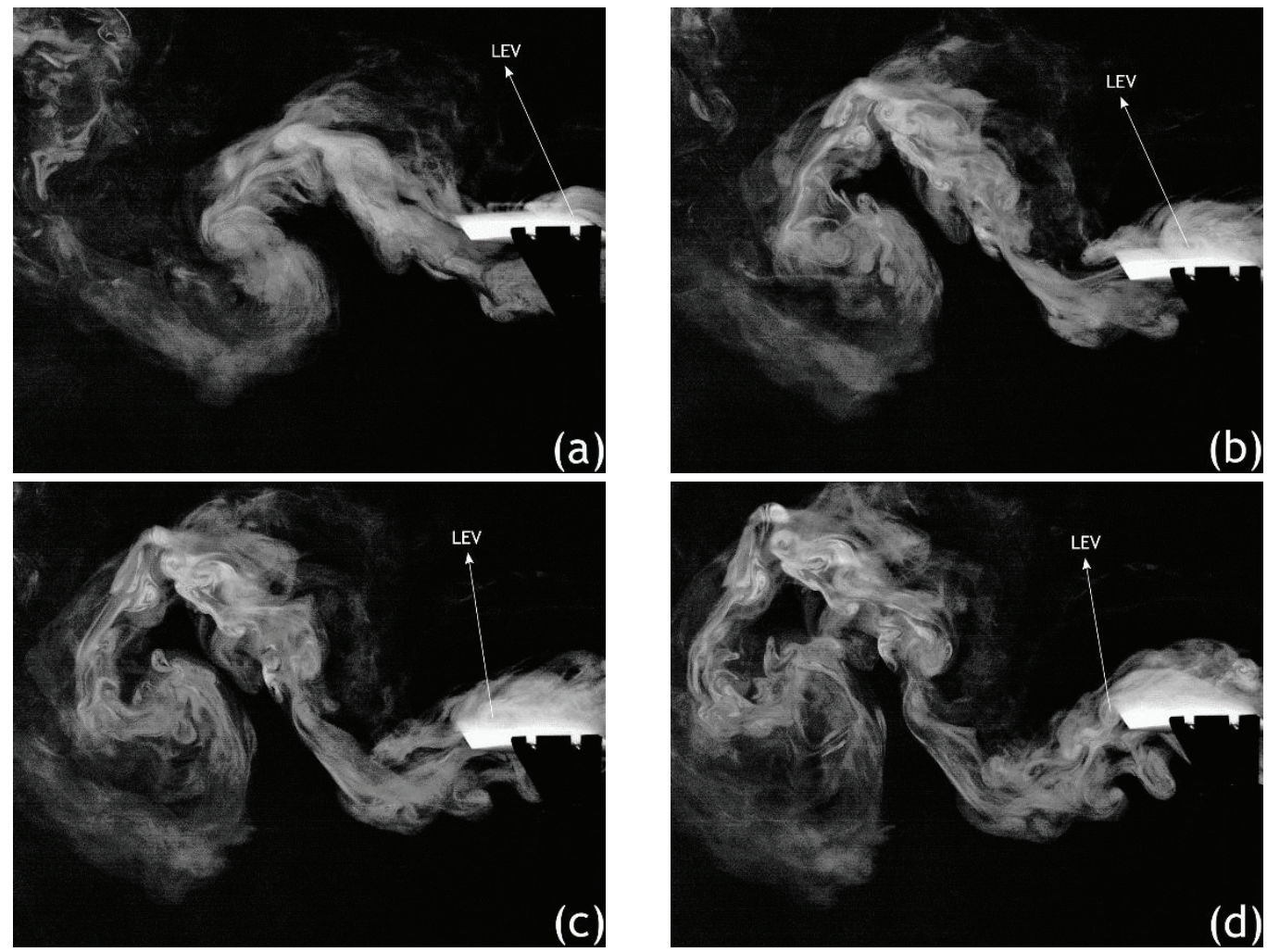

Figure 6: LEV phenomenon at $\operatorname{Re}=1,500$.
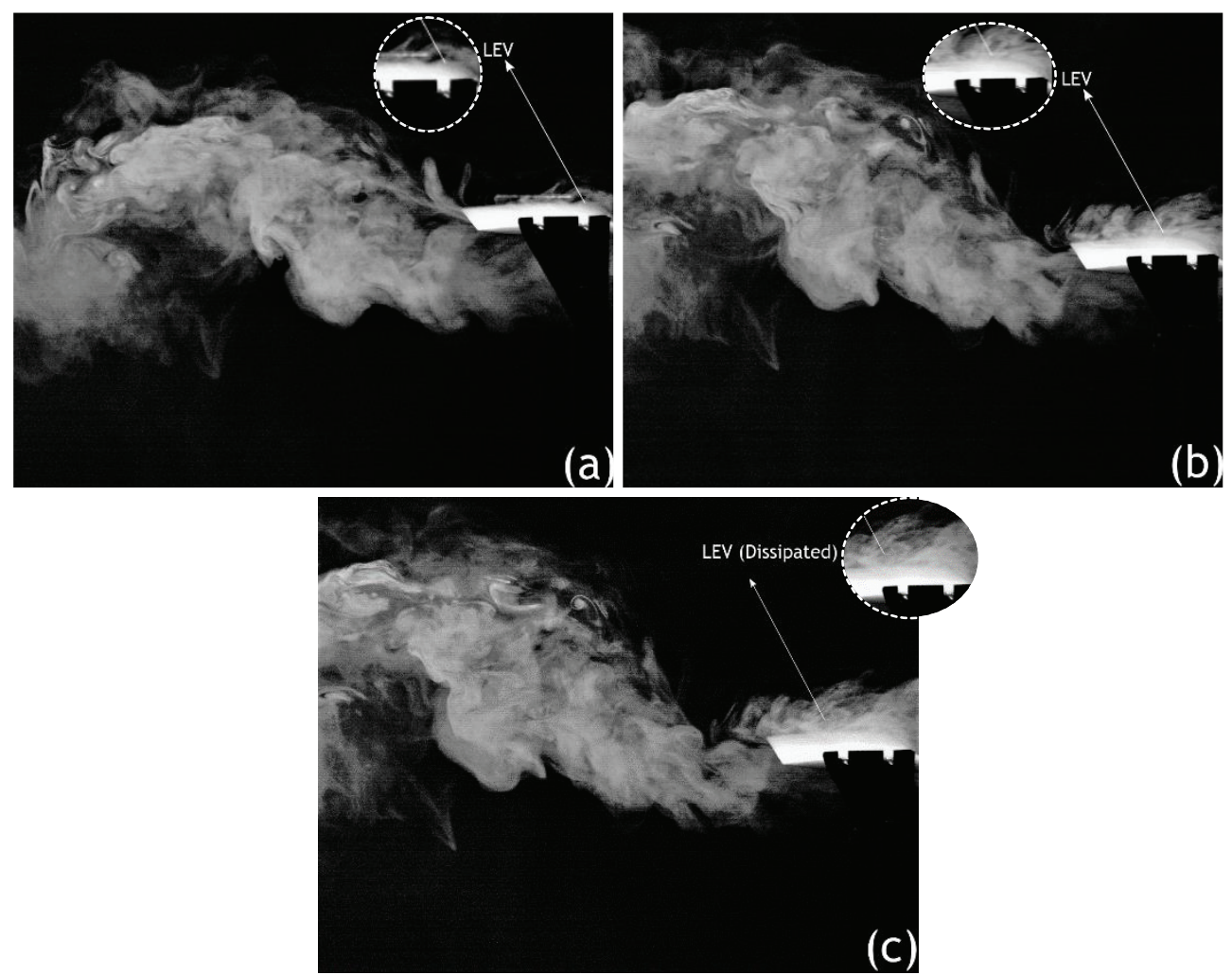

Figure 7: LEV phenomenon at $\operatorname{Re}=3,100$. 
wake and the time of the creation of the same structures. Since the velocity was changed, the reduced frequency inevitably changed, making the airfoil's inertial forces less impactful when compared to the flow field inertia. Also, an interesting symbiotic interaction of a LEV with a trailing-edge vortex was seen which may denounce that controlled flow separation may improve furthermore the aerodynamic performance of this system. The aerodynamics between flapping (nature flight) and conventional flight (fixed wings) differ substantially in two important subjects since on a fixed-wing, the air needs to remain attached and flows smoothly over the surface of an airfoil, and by contrast, the air over a flapping wing tends to be governed by unsteady effects, subject that should be intensively researched.

\section{Acknowledgments}

The present work was performed under the scope of the Aeronautics and Astronautics Research Center (AEROG) of the Laboratório Associado em Energia, Transportes e Aeronáutica (LAETA) activities and it was supported by Fundação para a Ciência e Tecnologia (FCT) through the project number UID/EMS/50022/2019.

\section{References}

[1] Lee J.-S.; Kim, C.; Kim, K.H., "Design of Flapping Airfoil for Optimal Aerodynamic Performance in Low-Reynolds," AIAA Journal, vol. 44, p. 1960-1972, 2006.

[2] Barata, J. M. M.; Manquinho, P. A. R.; Neves, F. M. S. P.; Silva, T. J. A., "Propulsion for Biological Inspired Micro-Air Vehicles (MAVs)", ICEUBI, International Conference on Engineering, Engineering for Society, Covilhã, 02/04 December 2015.

[3] Barata, J. M. M.; Manquinho, P. A. R.; Neves, F. M. S. P.; Silva, T. J. A., "Propulsion for Biological Inspired Micro-Air Vehicles (MAVs)," Open Journal of Applied Sciences, vol. 66, no. 1, pp. 7-15, 2016.

[4] Barata, J. M. M.; Manquinho, P. A. R., Neves, F. M. S. P.; "Comparative Study of Wing's Motion Patterns on Various Types of Insects on Resemblant Flight Stages," AIAA Science and Technology Forum 2015, Kissimmee, Florida, USA, 05/09 January 2015.

[5] Barata, J. M. M.; Silva, T. J. A.; Neves, F. M. S. P.; Silva, A. R. R, "Experimental Analysis of Forces During Take-Off of Birds," AIAA Science and Technology Forum and Exposition 2017, 09/13 January 2017, Grapevine, Texas, USA. 
[6] Barata, J. M. M.; Neves, F. M. S. P.; Manquinho, P. A. R.; Silva, A. R. R, "Biological Inspired Propulsion of Micro-Air Vehicles," 7th ECCOMAS, SMART 2015, Ponta Delgada, S. Miguel, Azores, Portugal, 3 - 6 June 2015.

[7] McMichael, J. M.; Francis, M. S., "Micro Air Vehicles - Toward a New Dimension in Flight”, http://www.fas.org/irp/program/collect/docs/mav_auvsi.htm (28.09.2019).

[8] Petricca, L.; Ohlckers, P.; Grinde, C., "Micro- and Nano-Air Vehicles: State of the Art", International Journal of Aerospace Engineering, Vol. 2011, Article ID 214549, 17 pages.

[9] Hylton, T.; Martin, C.; Tun, R.; Castelli, V., "The DARPA Nano Air Vehicle Program," 50th AIAA Aerospace Sciences Meeting Including the New Horizons Forum and Aerospace Exposition, 09-12 January 2012, Nashville, Tennessee, USA.

[10] Knoller, R., "Die Gesetze des Luftwiderstandes," Flug- und Motortechnik (Wien), vol. 3, no. 21, pp. 1-7, 1909.

[11] Betz, A., "Ein Beitrag zur Erklaerung des Segelfluges," Zeitschrift fur Flugtechnik und Motorluftschiffahrt, Vol. 3, pp. 269-272, 1912.

[12] Lai, J.C.S.; Platzer, M. F., "Jet characteristics of a plunging airfoil," AIAA Journal, vol. 37, no. 12, pp. 1529-1537, 1999. 6, 16, 19

[13] Lewin, G. C.; Haj-Hariri, H., "Modelling thrust generation of a two-dimensional heaving airfoil in a viscous flow, " Journal of Fluid Mechanics, vol. 492, p. 339362, 2003. 6

[14] Young, J., "Numerical simulation of the unsteady aerodynamics of flapping airfoils," Ph.D. dissertation, The University of New South Wales, Australian Defence Force Academy, 2005.

[15] J. Young, J.; Lai, J. C. S., "Vortex lock-in phenomenon in the wake of a plunging airfoil," AIAA Journal, vol. 45, no. 2, pp. 485-490, 2007. 6, 10, 23

[16] Taylor, R. L. N. G. K.; Thomas, A. L. R., "Flying and swimming animals cruise at a Strouhal number tuned for high power efficiency," Nature (London), vol. 425, pp. 707-711, 2003. 7

[17] Camacho, E. A. R., "Numerical Analysis of a Plunging NACA0012 Airfoil," Master's Dissertation, Universidade da Beira Interior, Covilhã, Portugal, 2019. 